\title{
Diagnostic Utility of Immunohistochemical Staining for p63, a Sensitive Marker of Prostatic Basal Cells
}

\author{
Michael H. Weinstein, M.D., Ph.D., Sabina Signoretti, M.D., Massimo Loda, M.D. \\ Department of Pathology, Brigham and Women's Hospital (MHW, ML) and the Dana Farber Cancer \\ Institute (ML, SS), Boston, Massachusetts
}

Diagnostically reliable identification of prostatic basal cells has depended on staining for high molecular weight cytokeratin. The diagnosis of malignancy is often based on the absence of basal cells. False-negative staining is occasionally observed. Thus, a second method of identifying basal cells might prove useful. Selective expression of p63, a homologue of p53, has been demonstrated in prostatic basal cells. We investigated the diagnostic utility of p63 staining in 70 consecutive specimens for which the differential diagnosis included prostatic adenocarcinoma: 68 needle biopsies and 2 transurethral resection blocks. High molecular weight cytokeratin staining was the gold standard when material was available. A total of 61 specimens were diagnosed as carcinoma, 4 as atrophy, 2 as highgrade prostatic intraepithelial neoplasia, 2 as unclassified collections of benign glands, and 1 as carcinoma versus high-grade prostatic intraepithelial neoplasia. Sections mounted on charged slides were used for p63 staining for 14 specimens. Sections previously hematoxylin and eosin stained on uncharged slides were used for 56 specimens. In every case in which there was successful p63 staining (55 specimens), basal cells in benign lesions were properly marked and other cell types were not stained. Uninformative staining in the remaining 15 specimens was due to failure of tissue adherence in 14 specimens in which sections were on uncharged slides and, in 1 specimen, to poor positive internal control staining of benign glands. Thus, p63 stain-

\footnotetext{
Copyright (C) 2002 by The United States and Canadian Academy of Pathology, Inc.

VOL. 15, NO. 12, P. 1302, 2002 Printed in the U.S.A.

Date of acceptance: September 3, 2002.

This work was supported in part by the Gelb Center for Translational Research of the Lank Center for Genitourinary Oncology, Dana Farber Cancer Center, Boston, Massachusetts. It was also supported by a National Institutes of Health grant (R01-CA81755), by Novartis and BarrWeaver Investigator grants, and by a CaPCURE Award (all to ML); and by a Hershey Prostate Cancer/Survivor Walk Award, a CaPCURE Award, and a Department of Defense grant (DAMD17-01-1-0051; all to SS). Address reprint requests to: Michael H. Weinstein, M.D., Ph.D., Department of Pathology, Wake Medical Center, 3000 New Bern Avenue, Raleigh, NC 27610; e-mail: mweinstein@wakemed.org; fax: 919-350-8232.

DOI: 10.1097/01.MP.0000038460.95912.6E
}

ing was informative in 55 of 56 specimens (98\%) for which there was material for examination. No case with satisfactory p63 and high molecular weight staining showed disagreement between the two stains. An additional group of 21 transurethral resection specimens was stained (p63 and high molecular weight cytokeratin). There was less falsenegative staining for $\mathrm{p} 63$ compared with the case of high molecular weight cytokeratin. No false-positive staining was seen. We conclude that p63 staining is at least as sensitive and specific for the identification of basal cells in diagnostic prostate specimens as is high molecular weight cytokeratin staining.

KEY WORDS: Basal cell, p63, High molecular weight cytokeratin, Prostate cancer.

Mod Pathol 2002;15(12):1302-1308

The knowledge that basal cells are invariably absent from the malignant glands of prostatic adenocarcinoma and the ability of immunohistochemical staining for high molecular weight cytokeratin to detect basal cells have proven to be diagnostically invaluable (1-4). This is particularly true for small foci of carcinoma commonly seen in needle biopsy specimens. From a biological perspective, it has been postulated that the basal cells represent the reserve cell compartment within the prostatic epithelium (5-8) and that interruption and loss of the basal cell layer are important steps in the genesis of invasive carcinoma from the putative precursor lesion, prostatic intraepithelial neoplasia (9). In practice, failure of staining for high molecular weight cytokeratin to demonstrate the presence of any basal cells within a collection of glands does not guarantee that those glands represent carcinoma. However, the presence of clearly identifiable basal cells in a gland or duct does preclude the diagnosis of carcinoma for that structure.

Expression of a recently cloned homologue of the tumor suppressor gene, p53, has been shown in the basal cell component of epithelium from a variety of tissues, including prostatic epithelium $(10,11)$. This gene, p63, appears to be quite important in the 
development of epithelial tissues in many organs $(12,13)$ and may be essential for the maintenance of epithelial stem cell populations. It has been shown that p63(-I-) mice fail to develop prostate glands (14). We have previously reported that in humans, prostatic adenocarcinoma is negative for p63 by immunohistochemistry in radical prostatectomy specimens $(14,15)$. This last observation suggests that staining for p63 may have diagnostic utility in differentiating benign prostatic epithelial structures from prostate cancer.

The goal of this study was primarily to test the diagnostic utility of immunohistochemical staining for p63 in the identification of prostate cancer. Staining for high molecular weight cytokeratin functions quite well for the purpose of differentiating benign prostatic epithelial structures from carcinoma. However, the immunohistochemical result supporting the diagnosis of prostate cancer is a negative one, and there are circumstances in which an ancillary antibody might prove useful. One example might be a situation in which the antigenicity of the tissue has been compromised by handling or processing and in which staining for high molecular weight cytokeratin fails to demonstrate basal cells in both identifiably benign and possibly malignant epithelium (i.e., there is poor positive internal control staining for high molecular weight cytokeratin). We have previously seen such instances in which antigenicity for p63 is preserved. Thus, a secondary, convenient means of identifying basal cells might be diagnostically useful, particularly in needle biopsy and transurethral resection (TURP) specimens. We tested the accuracy of staining for p63 in differentiating between benign lesions and prostate cancer in a series of diagnostic specimens (needle biopsies and TURP specimens) that contained at least one collection of glands for which a diagnosis of carcinoma was considered. We tested the results of p63 staining against the gold standard of high molecular weight cytokeratin staining when sufficient material was available.

\section{MATERIALS AND METHODS}

The investigation was confined to study of prostate needle biopsies and TURPs. An electronic search of the records of the Department of Pathology of the Brigham and Women's Hospital (BWH) was performed to identify these types of specimens dating from October 22, 1999 to April 25, 2000. Only those cases for which a diagnosis of carcinoma either was definitively made or was considered, based on the pathology report, were retrieved. Specimens (individual paraffin-embedded blocks) were ultimately included in the study if there was sufficient material so that sections could be stained for p63. Cases referred from outside institutions for which such material was available and a single additional, diagnostically challenging $\mathrm{BWH}$ case dating from 2001 were also included. The cases were chosen in this nearly consecutive fashion to obtain a representation of problems seen in daily practice. All available hematoxylin and eosin ( $\mathrm{H} \& \mathrm{E})$-stained and immunohistochemically stained material was used for diagnostic purposes in this study.

An additional collection of TURP specimens was included. This collection consisted of 21 blocks originating from 13 patients. These were consecutive cases for which material was available. For each case, the first one or two blocks that contained significant amounts of glandular prostatic tissue by review of the original $\mathrm{H}$ \& E-stained material were chosen for study. Sections were cut and stained for p63 and high molecular weight cytokeratin.

A secondary goal was to ascertain whether staining for p63 could be adequately performed on destained, uncharged slides that originally had been stained with $\mathrm{H} \& \mathrm{E}$. Thus, slides from cases for which there was no unstained material available either already on charged slides or in the tissue blocks were used in this investigation. This was done only when there were extra H \& E-stained slides with diagnostic material and permission was obtained from the referring institution.

When material was available, sections were also stained for high molecular weight cytokeratin (61 needle biopsies and 2 TURP specimens). This was done on sections placed on charged slides when possible (56 needle biopsies, 2 TURP). When this was not possible, staining for high molecular weight cytokeratin was performed on extra $\mathrm{H}$ \& E-stained slides that were first destained, when they were available (5 needle biopsies).

\section{Destaining of $\mathrm{H} \& \mathrm{E}-$ Stained Slides}

$\mathrm{H} \& \mathrm{E}$-stained slides were destained before immunohistochemical staining by rehydration followed by immersion in acid alcohol for a time sufficient to remove the $\mathrm{H} \& \mathrm{E}$ staining (approximately $20 \mathrm{~s})$.

\section{Immunohistochemical Staining for p63}

When additional sections were cut for p63 staining, they were cut at $5-\mu \mathrm{m}$ thickness and were placed on charged slides. The sections were deparaffinized and rehydrated. $\mathrm{H} \& \mathrm{E}$-stained slides were first destained as described above.

All slides stained for p63 were microwaved for 15 minutes in $10 \mathrm{mmol} / \mathrm{L}$ citrate buffer, $\mathrm{pH} 6.0$ (BioGenex, San Ramon, CA) in a 750-W oven. The slides were allowed to cool at room temperature for 30 minutes. The 4A4 anti-p63 antibody (10; gift from 
the laboratory of Dr. Frank McKeon, Harvard Medical School, Boston, MA), which recognizes all six p63 isotypes, was applied (1:50 dilution) at room temperature for 2 hours in an automated stainer (Optimax Plus 2.0 basal cell; BioGenex, San Ramon, CA). Detection steps were also performed using the automated stainer with the MultiLink-HRP kit (BioGenex). Peroxidase activity was localized using 3,3 diaminobenzidine or 3,3 diaminobenzidine-nickel chloride. Standardized development time periods allowed accurate comparison of all samples.

\section{Immunohistochemical Staining for High Molecular Weight Cytokeratin}

Staining for high molecular weight cytokeratin was performed using the $34 \beta \mathrm{E} 12$ antibody (DAKO, Carpinteria, CA) at a dilution of 1:250. Protease digestion (Sigma, St. Louis, MO) was done for 20 minutes before application of the primary antibody, and the Envision Developing System (DAKO) was used for visualization.

\section{Definition of Informative p63 Staining}

The H \& E-, p63-, and high molecular weight cytokeratin-stained slides were examined together for each case. An assessment of whether p63 staining was informative was made using high molecular weight cytokeratin staining as the gold standard, when available. Staining for p63 was considered informative when the absence of basal cells was clearly demonstrated in lesions that had been diagnosed as malignant, sometimes with the help of high molecular weight cytokeratin staining. Good internal positive control staining was required. Staining was also considered informative when basal cell nuclei were decorated in epithelium that had been diagnosed as benign.

\section{RESULTS}

A total of 70 specimens were identified, originating from 47 patients. These included one TURP ( 2 paraffin-embedded blocks) and 68 needle biopsy specimens. The final diagnoses on the glandular foci in question included 61 foci with definitive diagnoses of carcinoma, 4 diagnoses of atrophy, 2 diagnoses of high-grade prostatic intraepithelial neoplasia (HGPIN), 2 foci of otherwise unclassifi- able close-packed benign glands, and a single case in which the differential diagnosis remained between high-grade PIN and high-grade PIN with carcinoma.

Categorization of cases by final diagnosis and by whether p63 staining was informative is given in Table 1. p63 staining performed on charged slides was informative in 13 of 14 cases (93\%). Staining performed on noncharged slides was informative in 42 of 56 cases $(75 \%)$. In no case with good positive internal control staining for p63 was there a false negative. That is, basal cells in the glandular foci in question were highlighted in every case. Nor were any cases of spurious staining of nonbasal cells seen (Figs. 1A-G).

Staining for p63 failed to be informative in 15 specimens. In 14 of these (93\%), the reason was that the tissue failed to adhere to the slide during the antigen retrieval step. The one remaining case, in which the focus in question consisted of atrophy, showed clear evidence of poor internal positive control staining of basal cells in clearly benign glands. The staining in this case was, therefore, deemed uninterpretable. In no case did the results of p63 staining conflict with the assessment of the $\mathrm{H} \& \mathrm{E}-$ and high molecular weight cytokeratinstained material.

A greater fraction of specimens in which p63 staining was performed on charged slides was informative $(13 / 14,93 \%)$, compared with cases in which $\mathrm{H}$ \& E-stained slides were destained and then stained for p63 $(42 / 56,75 \%)$. This was due to a greater number of sections in the latter group failing to adhere to the slide during antigen retrieval (Table 2).

Staining for high molecular weight cytokeratin was performed on tissue from 63 blocks (61 needle biopsy specimens, 2 from a single TURP). In 6 specimens, there was poor internal positive control staining for high molecular weight cytokeratin. Three of these were needle biopsies for which staining was performed on destained slides that were previously stained with $\mathrm{H}$ \& E, and one was a needle biopsy for which an unstained section was available. The other two blocks were from the TURP, for which unstained sections were available. In 4 of these 6 specimens, there was better positive internal control staining for p63 than for high molecular weight cytokeratin. One case showed similarly poor

TABLE 1. Numbers of Specimens for which p63 Staining Was Informative by Final Diagnosis

\begin{tabular}{|c|c|c|c|c|c|c|}
\hline \multirow{2}{*}{$\begin{array}{l}\text { p63 Staining } \\
\text { Informative }\end{array}$} & \multicolumn{5}{|c|}{ Final Diagnoses } & \multirow[b]{2}{*}{ Tota } \\
\hline & Carcinoma & Atrophy & HGPIN & $\begin{array}{l}\text { Benign, Close- } \\
\text { Packed Glands }\end{array}$ & $\begin{array}{c}\text { Carcinoma versus } \\
\text { HGPIN }\end{array}$ & \\
\hline Yes & 48 & 3 & 1 & 2 & 1 & 55 \\
\hline No & 13 & 1 & 1 & 0 & 0 & 15 \\
\hline Total & 61 & 4 & 2 & 2 & 1 & 70 \\
\hline
\end{tabular}



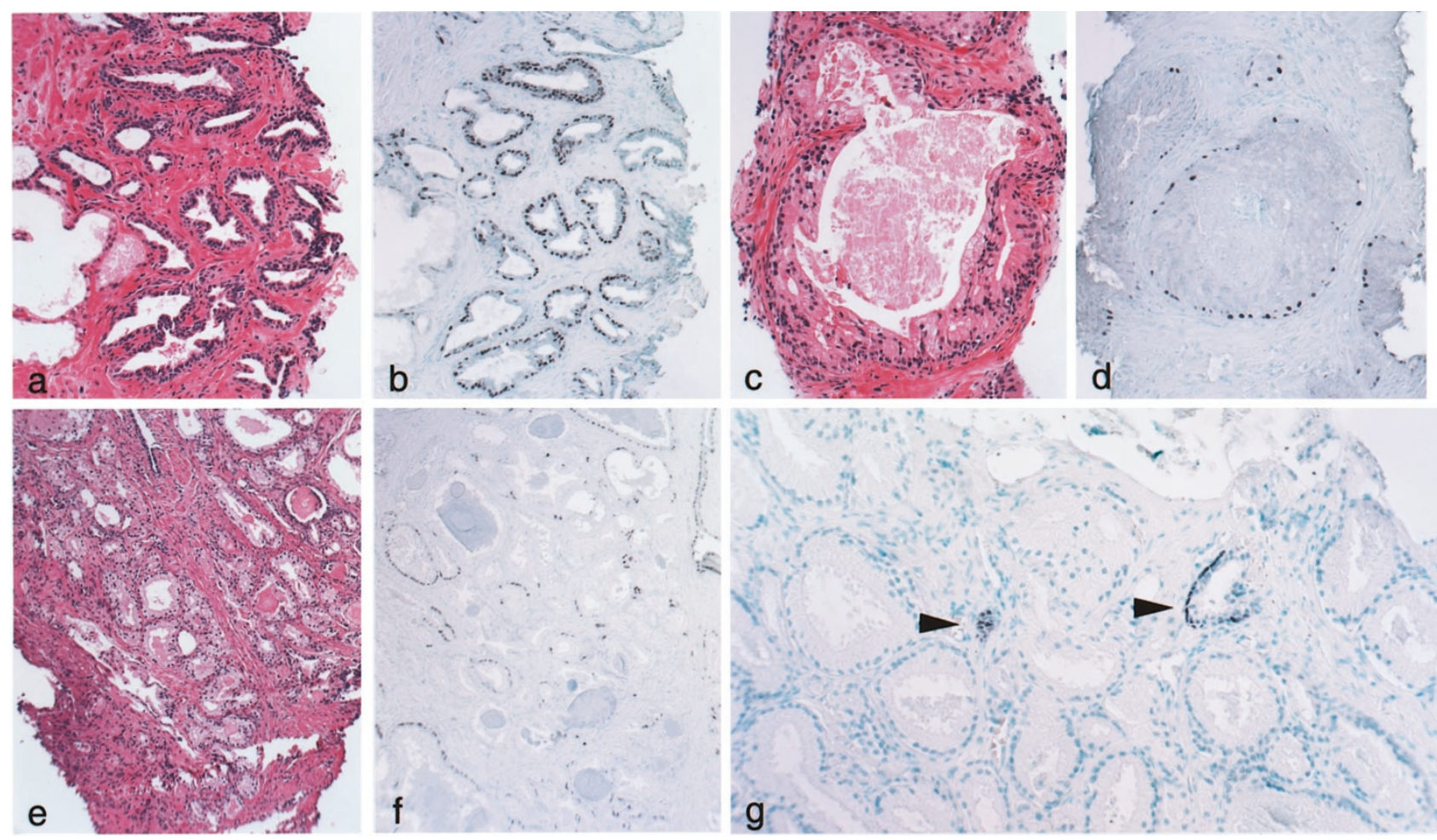

FIGURE 1. Representative types of glandular lesions stained. Atrophy: A, H \& E-stained section and B, section stained for p63. High-grade prostatic intraepithelial neoplasia: C, H \& E-stained section and D, section stained for p63. Atypical adenomatous hyperplasia (adenosis) in a TURP specimen: E, H \& E-stained section and F, section stained for p63. Adenocarcinoma: G, p63 staining showing the malignant glands to be devoid of basal cells, but basal cells are highlighted in two entrapped benign glands (arrowheads).

TABLE 2. Numbers of Specimens for which p63 Staining Was Informative

\begin{tabular}{lccc}
\hline Charged Slides for p63 Staining & $\begin{array}{c}\text { Informative, } \\
n(\%)\end{array}$ & $\begin{array}{c}\text { Not } \\
\text { Informative, } \\
n(\%)\end{array}$ & Total \\
\hline Yes & $13(93)$ & $1(7)$ & 14 \\
No & $42(75)$ & $14(25)$ & 56 \\
$\quad$ Tissue adhered to slide & $55(98)$ & $1(2)$ & 56 \\
\hline
\end{tabular}

staining for p63 as for high molecular weight cytokeratin, and in one case the section for p63 staining failed to adhere.

In addition to the index glandular foci, at least 20 foci of atrophy were identified that did not present any diagnostic challenge. In every case with good positive internal control staining of basal cells for p63, the foci of atrophy showed prominent basal cell staining.

An additional collection of TURP specimens was included. It consisted of 21 blocks originating from 13 patients. In 8 blocks there was at least one focus of benign glands that failed to show high molecular weight cytokeratin-positive basal cells. Basal cells positive for p63 were seen in every one of these foci, some of which showed significant cautery artifact. No clusters of architecturally and cytologically benign glands without p63-positive basal cells were seen, although scattered single p63-negative benign glands could be found. In one block, a focus of adenocarcinoma was found. It was negative for both p63 and high molecular weight cytokeratin.

\section{DISCUSSION}

In 1953, Totten et al. (16) observed that basal cells were invariably lacking in prostatic adenocarcinoma. They also stated that basal cells were not always present in benign prostatic epithelium. This latter statement is indicative of how inconspicuous basal cells can be on $\mathrm{H}$ \& E-stained material. It was not until the advent of immunohistochemical staining for cytokeratins that are preferentially expressed in basal cells that the diagnostic utility of the Totten et al. (16) observation became fully appreciated. In 1985, Brawer et al. (1) clearly outlined the manner in which staining for high molecular weight cytokeratin could be used to distinguish a variety of benign and potentially preneoplastic processes from invasive carcinoma. Widespread use of serum PSA screening and 18-gauge needle biopsy technique has led to a large increase in the number of biopsies, and interpretation of small, diagnostically challenging collections of atypical glands is now a routine part of surgical pathology practice. Immunohistochemical staining for high molecular weight cytokeratin has become a powerful tool in rendering definitive diagnoses in a significant fraction of these cases (2-4). 
With almost any immunohistochemical stain, reliable preservation of accessible epitope is not always assured in formalin-fixed, paraffin-embedded tissue. This has been demonstrated to be true for high molecular weight cytokeratin, as detected with the $34 \beta$ E12 antibody (17-19). Occasional failure of staining is of great significance regarding the use of antibodies to high molecular weight cytokeratin in the detection of prostate cancer because the diagnostic finding is a negative result (i.e., absence of staining). Studies have shown false-negative staining in as many as $88 \%$ of TURP specimens (18) and in up to $5 \%$ of needle biopsy specimens (19). Modification of staining technique can improve on these results (17-19). However, a second reliable method for detecting basal cells would likely be of use in difficult cases in which there is suspicion that high molecular weight cytokeratin staining is unreliable.

The recently cloned gene, $\mathrm{p} 63$, is a homologue of the tumor suppressor gene, p53 $(10,20-22)$. It is expressed in the basal cell component of the epithelium in a variety of human tissues and appears to be important in epithelial embryogenesis (10, 11). Homozygous knockout mice fail to develop prostate glands (14) and also show agenesis of squamous epithelia as well as failure to form breast, salivary, and lachrymal gland tissue $(12,13)$. The continued expression of p63 in adult tissues suggests that it may be important in the maintenance of these epithelial structures and that the basal cells of normal prostatic epithelium comprise the generative reserve population that may continually replace lost secretory epithelial cells (5-8).

It has already been shown that immunohistochemical staining for p63 selectively labels basal cells in prostatectomy specimens (14). A study by Parsons et al. (15) included needle biopsies, as well as prostatectomy specimens. Neither secretory epithelial cells nor the neuroendocrine cells of prostatic epithelium were found to express this protein. We performed the present study to assess the diagnostic utility of p63 staining and concentrated our investigation on cases with specific glandular foci of diagnostic concern. A nearly consecutive series of 70 diagnostic specimens (2 TURP, 68 needle biopsies) for which the diagnosis of carcinoma was initially included in the differential diagnosis was chosen for study. Negative p63 staining was considered informative when it failed to mark any cells in a glandular focus diagnosed as malignant and good positive internal control staining was present. Positive staining was considered informative when p63 staining highlighted the presence of basal cells in a glandular focus that was diagnosed as benign or as representing high-grade prostatic intraepithelial neoplasia. Staining for high molecular weight cyto- keratin was performed as the gold standard for comparison, when possible.

A secondary goal was to assess the reliability with which p63 staining could be performed on destained tissue mounted on uncharged slides that had previously been stained with $\mathrm{H} \& \mathrm{E}$.

In every case in which diagnostic material was successfully stained for p63 and good positive internal control staining was seen, p63 staining was informative (Figs. 1A-G). That is, no staining was seen in malignant glands, and the basal cells in benign glands were stained. Of 70 specimens, 15 (21\%) failed to be informative. All but one (14/15) of these uninformative cases were ones in which an attempt was made to perform staining on uncharged slides and the tissue failed to adhere. The single remaining specimen was one in which there was poor internal positive control staining of basal cells in adjacent, clearly benign epithelium. Thus, 55 of 56 (98\%) cases in which there was stained material for examination were informative. In addition to the 70 specific glandular foci in question in the entire group of specimens, at least 20 foci of atrophy were identified in specimens with good positive internal control staining (Fig. 1A-G). p63 staining highlighted the presence of basal cells in every one of these foci of atrophy.

The results for p63 staining compare favorably with the results for high molecular weight cytokeratin staining, which was performed on 63 specimens (Fig. 2A-D); high molecular weight cytokeratin staining was uninformative in 6 of these specimens: two specimens (blocks) from a single TURP, three specimens for which staining was performed on destained slides, and one specimen for which an unstained section mounted on a charged slide was available. In all of these six specimens, there was poor positive internal control staining. In four of the six specimens, there was better staining for p63. One case showed similarly poor positive internal control staining for p63 as for high molecular weight cytokeratin, and in one case the tissue for p63 staining failed to adhere to the slide. In no case in which both stains were technically satisfactory was there disagreement between p63 staining and high molecular weight cytokeratin staining.

An additional collection of TURP specimens was included. In 8 of 21 blocks, there was at least one focus of benign glands that failed to show high molecular weight cytokeratin-positive basal cells. p63-positive basal cells were seen in every one of these foci, some of which showed significant cautery artifact (Fig. 2A-B). No clusters of architecturally and cytologically benign glands without p63positive basal cells were seen, although scattered single p63-negative benign glands could be found. In one block, a focus of adenocarcinoma was 

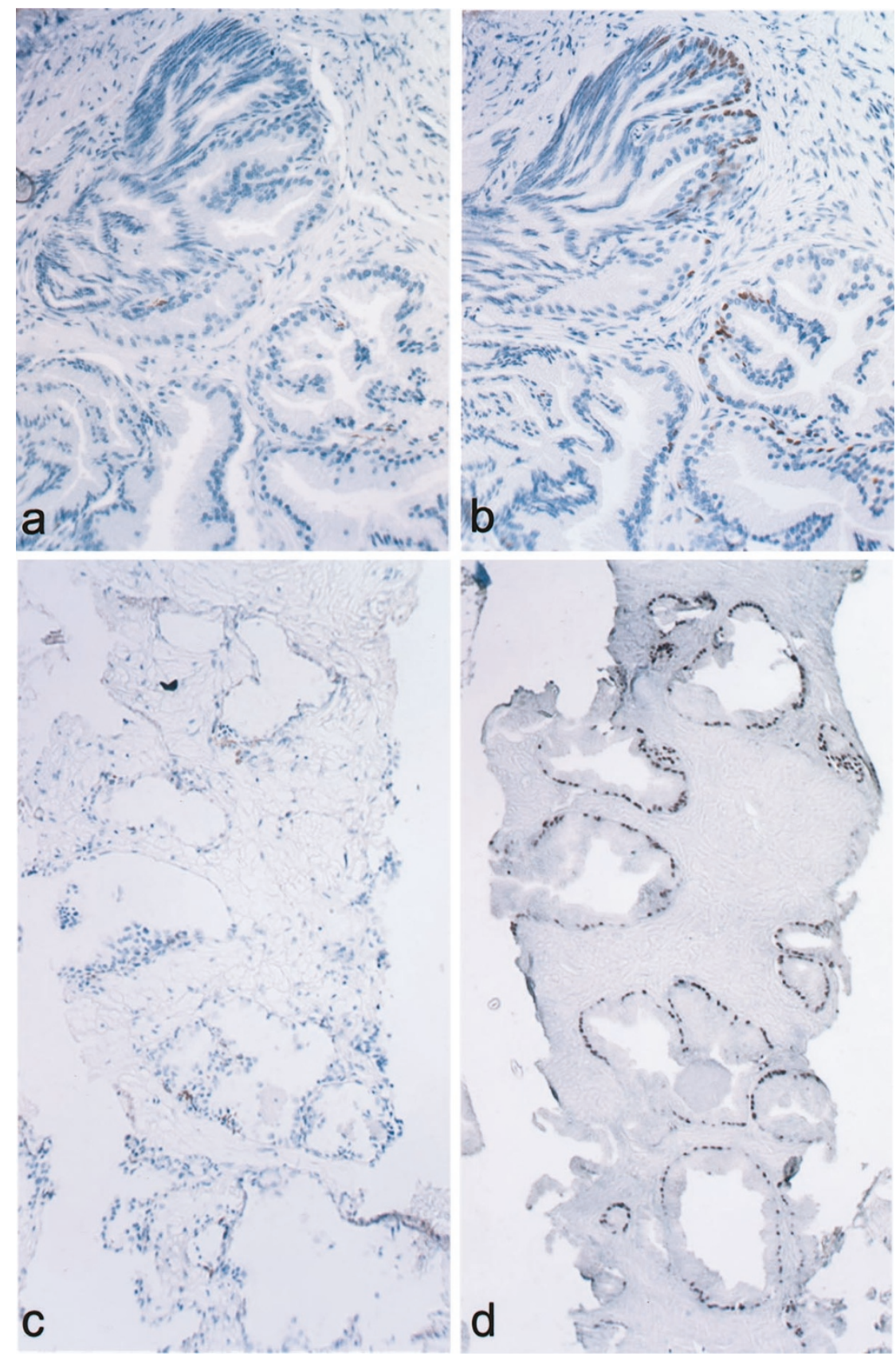

FIGURE 2. Two examples of staining for p63 being more sensitive that HMWCK staining. Benign prostatic tissue in a TURP specimen with cautery artifact: A, section stained for high molecular weight cytokeratin and B, section stained for p63. Note lack of high molecular weight cytokeratin staining of basal cells in (A) and clusters of basal cells highlighted by nuclear p63 staining in (B). Benign glands in a needle biopsy: comparison of high molecular weight cytokeratin staining (C) and p63 staining (D) in a case with poor positive internal control staining for high molecular weight cytokeratin.

present. It was negative for both p63 and high molecular weight cytokeratin.

We conclude that p63 staining is sensitive in identifying basal cells in benign lesions and will not lead to false-positive diagnoses of malignancy in needle biopsies of the prostate. Moreover, staining of cells other than basal cells was not observed, indicating that use of this stain would not lead to false-negative diagnoses. Therefore, p63 appears to have diagnostic utility similar to that of high molecular weight cytokeratin staining. TURP speci- mens, in particular, commonly show poor high molecular weight cytokeratin staining of basal cells in benign glands, especially in areas with cautery artifact (18). This type of specimen, however, appears to show better preservation of p63 antigenicity.

We found a poor rate of tissue preservation with tissue mounted on uncharged slides using our microwave method of antigen retrieval before p63 staining. That is, the tissue failed to adhere for $\mathrm{p} 63$ staining for $25 \%$ of these specimens. In our laboratory, protease pretreatment as used for high molec- 
ular weight cytokeratin staining is more gentle and is less likely to result in loss of tissue when sections on uncharged slides are all that are available. Thus, in this circumstance, high molecular weight cytokeratin staining may be superior.

In summary, we found that immunohistochemical p63 staining is diagnostically reliable in identifying basal cells in prostatic needle biopsies and TURP specimens and compares favorably with high molecular weight cytokeratin staining. In TURP specimens in which cautery artifact can impair the ability to detect high molecular weight cytokeratin, staining for p63 appears superior. Finally, the microwave antigen retrieval used in this study resulted in a greater rate of tissue loss with sections mounted on uncharged slides than did the protease digestion used with high molecular weight cytokeratin staining.

\section{REFERENCES}

1. Brawer KB, Peehl DM, Stamey TA, Bostwick DG. Keratin immunoreactivity in the benign and neoplastic human prostate. Cancer Res 1985;45:3663-7.

2. Gaudin PB, Reuter VE. Benign mimics of prostatic adenocarcinoma on needle biopsy. Anat Pathol 1997;2:111-34.

3. Hedrick L, Epstein JI. Use of keratin 903 as an adjunct in the diagnosis of prostate carcinoma. Am J Surg Pathol 1989;13.

4. Wojno KJ, Epstein JI. The utility of basal cell-specific anticytokeratin antibody (34 beta E12) in the diagnosis of prostate cancer. A review of 228 cases. Am J Surg Pathol 1995;19: 251-60.

5. Robinson EJ, Neal DE, Collins AT. Basal cells are progenitors of luminal cells in primary cultures of differentiating human prostatic epithelium. Prostate 1998;37:149-60.

6. Verhagen AP, Aalders TW, Ramaekers FC, Debruyne FM, Schalken JA. Differential expression of keratins in the basal and luminal compartments of rat prostatic epithelium during degeneration and regeneration. Prostate 1988;13:25-38.

7. Jones EG, Harper ME. Studies on the proliferation, secretory activities, and epidermal growth factor receptor expression in benign prostatic hyperplasia explant cultures. Prostate 1992;20:133-49.

8. Peehl DM, Leung GK, Wong ST. Keratin expression: a measure of phenotypic modulation of human prostatic epithelial cells by growth inhibitory factors. Cell Tissue Res 1994;277:11-8.
9. Bostwick DG, Brawer KB. Prostatic intra-epithelial neoplasia and early invasion in prostate cancer. Cancer 1987;59:78894.

10. Yang A, Kaghad M, Wang Y, Gillett E, Fleming MD, Dotsch V, et al. p63, a p53 homolog at 3q27-29, encodes multiple products with transactivating, death-inducing, and dominant-negative activities. Mol Cell 1998;2:305-16.

11. Parsa R, Yang A, McKeon F, Green H. Association of p63 with proliferative potential in normal and neoplastic human keratinocytes. J Invest Dermatol 1999;113:1099-105.

12. Yang A, Schweitzer R, Sun D, Kaghad M, Walker N, Bronson RT, et al. p63 is essential for regenerative proliferation in limb, craniofacial and epithelial development. Nature 1999; 398:714-8.

13. Mills AA, Zheng B, Wang XJ, Vogel H, Roop DR, Bradley A. p63 is a p53 homologue required for limb and epidermal morphogenesis. Nature 1999;398:708-13.

14. Signoretti S, Waltregny D, Dilks J, Isaac B, Lin D, Garraway L, et al. p63 is a prostate basal cell marker and is required for prostate development. Am J Pathol 2000;157:1769-75.

15. Parsons JK, Gage WR, Nelson WG, De Marzo AM. p63 protein expression is rare in prostate adenocarcinoma: implications for cancer diagnosis and carcinogenesis. Urology 2001;58: 619-24.

16. Totten RS, Heinemann NW, Hudson PB, Sproul EE, Stout AP. Microscopic differential diagnosis of latent carcinoma of the prostate. Arch Pathol Lab Med 1953;55:131-41.

17. Iczkowski KA, Cheng L, Crawford BA, Bostwick DG. Steam heat with an EDTA buffer and protease digestion optimizes immunohistochemical expression of basal cell-specific antikeratin $34 \beta \mathrm{E} 12$ to discriminate cancer in prostatic epithelium. Mod Pathol 1999;12:1-4.

18. Multhaupt HAB, Fessler HT, Warhol MJ. Loss of highmolecular-weight cytokeratin antigenicity in prostate tissue obtained by transurethral resections. Arch Pathol Lab Med 2000;124:1764-7.

19. Varma M, Linden MD, Amin MB. Effect of formalin fixation and epitope retrieval techniques on antibody $34 \beta \mathrm{E} 12 \mathrm{immu}-$ nostaining of prostatic tissues. Mod Pathol 1999;12:472-8.

20. Osada M, Ohba M, Kawahara C, Ishioka C, Kanamaru R, Katoh I, et al. Cloning and functional analysis of human p51, which structurally resembles p53. Nat Med 1998;4:839-43.

21. Senoo M, Seki N, Sugano S, Watanabe M, Inuzuka S, Okamoto T, et al. A second p53-related protein, p73L, with high homology to p73. Biochem Biophys Res Commun 1998;248: 603-7.

22. Trink B, Ikami K, Wu L, Sriuranpong V, Jen J, Sidransky D. A new human p53 homologue. Nat Med 1998;4:747-8. 